\title{
Primary pulmonary botryomycosis: a late complication of foreign body aspiration
}

\author{
J M Tuggey, H S R Hosker, P DaCosta
}

\begin{abstract}
Primary pulmonary botryomycosis is a rare cause of haemoptysis and can enter the differential diagnosis of a mass on the plain chest radiograph. The case history is presented of a 63 year old man with botryomycosis which was initially thought to be a bronchial carcinoma. When the diagnosis was made several years later it was found to be secondary to persisting vegetable material in the bronchial tree following previous aspiration.

(Thorax 2000;55:1068-1069)
\end{abstract}

Keywords: botryomycosis; foreign body

Bollinger first described botryomycosis in 1870 in horse lung. ${ }^{1}$ Most of the reported cases have been cutaneous while visceral botryomycosis is relatively rare. We describe a case of pulmonary botryomycosis initially mistaken for bronchial carcinoma, believed to be secondary to foreign body aspiration several years previously.

\section{Case report}

A 63 year old man with longstanding mild asthma, obstructive sleep apnoea (OSA) for which he was receiving continuous nocturnal positive airways pressure (CPAP), and type II diabetes mellitus was admitted in early 1999 with a six month history of intermittent haemoptysis and mucopurulent sputum. $\mathrm{He}$ had suffered an episode of lobar pneumonia as

Department of Respiratory Medicine, Airedale General Hospital, Keighley, West Yorkshire BD20 6TD, UK

J M Tuggey H S R Hosker P DaCosta

Correspondence to: Dr H S R Hosker, Department of Respiratory Medicine, Airedale General Hospital, Keighley, West Yorkshire BD20 6TD, UK

Received 6 September 1999 Returned to authors 5 October 1999 Revised version received 3 December 1999 Accepted for publication 21 December 1999 a child. The patient had never smoked.

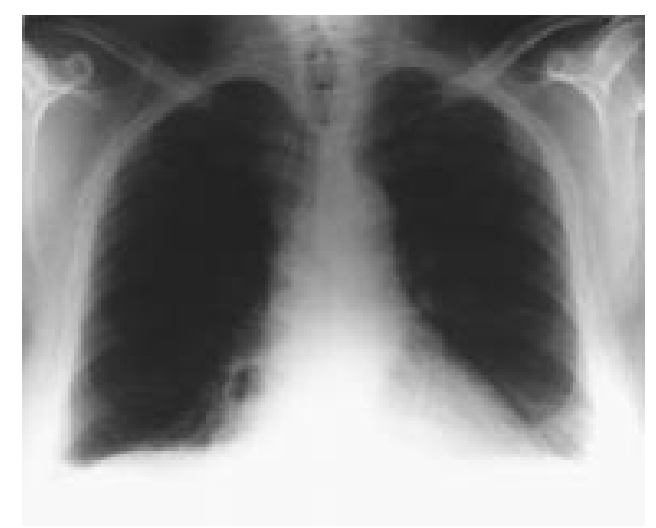

Figure 1 Chest radiograph showing an air fluid level adjacent to the right heart border.
He had first presented with haemoptysis in 1995. A routine chest radiograph and subsequent thoracic computed tomographic (CT) scan at that time confirmed the presence of a $3.5 \times 2 \times 2 \mathrm{~cm}$ soft tissue mass within the right lower lobe. The presence of a mass was confirmed by bronchoscopic examination. A biopsy specimen was not taken as the lesion was felt to be too vascular. A presumptive diagnosis of bronchial carcinoma was made. $\mathrm{He}$ was declined surgery on the basis of comorbidity (ischaemic heart disease, morbid obesity) and poor lung function (forced expiratory volume in one second $\left(\mathrm{FEV}_{1}\right) 1.2$ litres). The plan was therefore for observation with palliative radiotherapy if he became symptomatic. He continued with regular follow up with no change in the size of the mass radiographically.

On this admission, four years later, he was unwell with tachycardia and a temperature of $38^{\circ} \mathrm{C}$. The jugular venous pressure was raised and there was peripheral oedema. Coarse inspiratory crackles were evident in the right lower zone.

Laboratory investigations revealed a neutrophil leucocytosis (total white cell count $22.5 \times$ $10^{9} / 1$, neutrophils $\left.19.9 \times 10^{9} / 1(88 \%)\right)$. The plasma viscosity was 1.98 (normal range $1.50-$ 1.72).

Chest radiography revealed right lower zone consolidation but no mass lesion. There was, however, a cavity with an air fluid level adjacent to the right heart border (fig 1). Fibreoptic bronchoscopy performed the following week confirmed the presence of an endobronchial lesion almost occluding the right lower lobe bronchus (in the same location as in 1995). There were considerable mucopurulent secretions distal to this. Microscopic examination of the aspirate showed Gram stain positive cocci together with numerous pus cells. A biopsy specimen of the lesion revealed evidence of both botryomycosis and vegetative material, probably a peanut.

The patient was treated for 10 days with broad spectrum intravenous antibiotics (metronidazole, benzylpenicillin, and gentamicin). He made a good recovery and a follow up chest radiograph showed resolution of the lobar consolidation and reduction in the size of the air fluid opacity. At six months the patient remained well, with almost complete resolution of the radiographic appearances. 


\section{Discussion}

Botryomycosis is a bacterial infection that closely mimics infection by Actinomyces species. It is characterised by the presence of eosinophilic granules, usually in association with an infective focus. The granules comprise densely packed microorganisms surrounded by proteinaceous material, thought to be immunoglobulin. This histological pattern is referred to as the Splendore-Hoeppli phenomenon. ${ }^{2}$ The eosinophilic granules resemble the sulphur granules of actinomycosis but, when cultured, organisms such as Staphylococcus aureus, Escherichia coli, and Pseudomonas aeruginosa are grown.

Approximately 89 case reports of human botryomycosis have been reported, most of which $(75 \%)$ were cutaneous. ${ }^{3}$ Visceral botryomycosis, although well recognised, is relatively rare with few case reports in the literature. ${ }^{45}$

Previous reports suggest that the presence of a foreign body is required to provoke the granule formation of botryomycosis. ${ }^{5}{ }^{6}$ The patient could not recall having aspirated a peanut in the past. The size of the inoculum, virulence of the organism, and host defences are all believed to be cofactors in the bacterial formation of granules. There is also evidence that immunosuppression such as diabetes mellitus, chronic granulomatous disease, or HIV infection is an important cofactor. ${ }^{378}$ Our patient was a longstanding diabetic.

There are few case reports which detail the management of primary pulmonary botryomy- cosis. Most patients have been treated empirically with broad spectrum antibiotics, occasionally requiring surgery in unresolving cases. ${ }^{8}$

We would suggest that the original lesion seen on the chest radiograph in 1995 was the same as that seen in 1999 and predated treatment with CPAP. He had been treated by his general practitioner during this time for several chest infections associated with mucopurulent secretions. Chronic low grade infection surrounding a foreign body resulted in the botryomycosis lesion.

This case illustrates how botryomycosis can present as a mass on a chest radiograph and, as such, can mimic a primary bronchogenic carcinoma. We would suggest that, although rare, pulmonary botryomycosis should enter the differential diagnosis of haemoptysis or an apparent mass on chest radiography.

1 Bollinger O. Mycosis der Lunge beim Pferde. Virchow's Arch Pathol Anat 1870;49:583-6.

2 Dail DH, Hammer SP. Pulmonary pathology. New York: Springer Verlag, 1988: 160-2.

3 Katapadi K, Pujol F, Vuletin JC, et al. Pulmonary botryomycosis in a patient with AIDS. Chest 1996;109: $276-8$.

4 Multz AS, Cohen R, Azeuta V. Bacterial pseudomycosis: a rare cause of haemoptysis. Eur Respir F 1994;7:1712-3.

5 Bersoff-Matcha SJ, Roper CC, Liapis H, et al. Primary pulmonary botryomycosis: case report and review. Clin Infect Dis 1998;26:620-4.

6 Plaut A. Botryomycosis in man. Arch Pathol 1937;23:602-3.

7 Kimmelstiel P, Oden PW. Botryomycosis. Report of two Kimmelstiel P, Oden PW. Botryomycosis. Report of two
cases of intra-abdominal granuloma. Arch Pathol 1939;27: 313-9.

8 Brunken RC, Lichon-Chao N, van der Broek H. Immunologic abnormalities in botryomycosis: a case report with review of the literature. $\mathcal{F}$ Am Acad Dermatol 1983;9:42834 